\title{
28 Research Soure \\ Genomic analyses identify biological processes of miR-125 mediated breast cancer cells
}

Hongmei Guo

Wei Zhang

Guangqi Liu

Tingxiang Chang

Hanming Gu ( $\sim$ laygmp@gmail.com )

Research Article

Keywords:

Posted Date: December 30th, 2021

DOI: https://doi.org/10.21203/rs.3.rs-1214908/v1

License: (c) (1) This work is licensed under a Creative Commons Attribution 4.0 International License.

Read Full License 


\section{Abstract}

miRNAs contain suppressive and oncogenic properties to regulate the progression of cancers. The expression of miR-125b expression is reported to be consistently low in breast cancers. However, the function and mechanism of miR-125b are not fully understood in breast cancers. In our study, our objective is to identify the DEGs and biological processes in the miR-125b mediated breast cancer cells by analyzing the RNA-seq data. The GSE123358 dataset was produced by the Illumina HiSeq 2000 (Homo sapiens). The KEGG and GO analyses showed the mitochondria and endoplasmic reticulum oxidative phosphorylation pathways are the main processes in miR-125b mediated breast cancer. Moreover, we identified ten interactive molecules including UQCRQ, CALR, HNRNPU, ATP5G1, NDUFB11, UQCRH, HSP90B1, TGOLN2, SAP18, and XPOT. Thus, our study may benefit the treatment of breast cancer by using miR-125b.

\section{Introduction}

Breast cancer is a heterogeneous disease, which is the most common cancer in women ${ }^{1}$. Recently, treatments have been involved in more biologically-directed therapies to decrease the adverse effects ${ }^{2}$. Early breast cancer is considered curable due to the reason that cancer only spreads to the axillary lymph nodes $^{3}$. Improvements in multiple therapies contribute to enhancing chances for cure in most patients ${ }^{4}$. Conversely, metastatic diseases are not curable by using the available therapeutic methods ${ }^{5}$. For metastatic cancer, the main goals of therapy are to prolong survival and improve quality of life ${ }^{6}$.

Small RNAs are defined by their length and association with AGO family proteins, which are divided into three classes including miRNA, siRNA, and piRNA ${ }^{7}$. miRNA contains about 22 nucleotides and are created by RNase III proteins, Drosha, and Dicer ${ }^{8}$. miRNA functions as a guide by matching its target mRNAs, whereas AGO proteins function as translational repressors and mRNA decay ${ }^{9}$. The miRNA regulation involves multiple steps such as transcription and Drosha/Dicer procession ${ }^{10}$. Most protein-coding genes include at least one conserved miRNA-binding site, which may be directly regulated by miRNAs ${ }^{11}$.

miR-125 is an important mediator in cancers, which binds to the RNA-induced silencing complex (RISC) to degrade the target mRNAs or inhibit the translation through binding the $3^{\prime} U T R s^{12}$. miR-125 contains a synergistic effect with a similar miRNA cluster ${ }^{13}$. Moreover, miR-125 has the ability of IncRNA to repress the expression ${ }^{14}$.

In this study, we analyzed the effects of miR-125b on breast cancer cells by using the RNA-seq data. We figured out several DEGs and functional biological processes. We also introduced the gene enrichment and the protein-protein interaction (PPI) network to find the interacting molecules. The significant signaling found in our study will improve the treatment of breast cancer.

\section{Methods}




\section{Data resources}

Gene dataset GSE123358 was obtained from the GEO database. The data was produced by the Illumina HiSeq 2000 (Homo sapiens) (Special Histopathology Laboratory, National University of Singapore, Lvl 3, NUH Main Building, 5 Lower Kent Ridge Road, Kent Ridge, Singapore). The analyzed dataset includes 12 high miR-125b breast cancer cells $(\mathrm{H})$ and 9 low miR-125b breast cancer cells $(\mathrm{L})$.

Data acquisition and processing

The data were organized and conducted by $\mathrm{R}$ package as previously described ${ }^{15-17}$. We used a classical t-test to identify DEGs with $P<0.05$ and fold change $\geq 1.5$ as being statistically significant.

The Kyoto Encyclopedia of Genes and Genomes (KEGG) and Gene Ontology (GO)

KEGG and GO analyses were conducted by R package (clusterProfiler) and Reactome. $\mathrm{P}<0.05$ was considered statistically significant.

Protein-protein interaction (PPI) networks

The Molecular Complex Detection (MCODE) was used to construct the PPI networks. The significant modules were produced from constructed PPI networks. The pathway analyses were performed by using Reactome (https://reactome.org/), and $\mathrm{P}<0.05$ was considered significant.

\section{Results}

\section{Identification of DEGs by the impact of miR-125b on breast cancer cells}

To determine the mechanism of miRNA-125b on the transcriptome of human breast cancer cells, we analyzed the sorted cells with maximum and minimum miR-125b expression. A total of 48 genes were identified with the threshold of $P<0.05$. The top ten of up-and-down-regulated genes by the impact of miR-125b on cancer cells are identified by the heatmap and volcano plot (Figure 1). The top DEGs were indicated in Table 1.

\section{Enrichment analysis of DEGs by the impact of miR-125b on breast cancer cells}

To further analyze the mechanism of miR-125b impacted cancer cells, we analyzed the KEGG and GO enrichment (Figure 2). The top ten KEGG items include "Pathways of neurodegeneration - multiple diseases", "Oxidative phosphorylation”, "Protein processing in endoplasmic reticulum”, "Parkinson disease”, “Alzheimer disease”, “Non-alcoholic fatty liver disease”, “Diabetic cardiomyopathy”, “Chemical carcinogenesis-reactive oxygen species”, "Thermogenesis”, “Nucleocytoplasmic transport”. We then identified the top ten biological processes "Maintenance of location”, "Striated muscle tissue development”, “Maintenance of location in cell”, "Protein-containing complex localization”, "Ubiquitin-dependent ERAD pathway", "Mitochondrial electron transport, ubiquinol to cytochrome c”, 
"Protein folding in endoplasmic reticulum", "Lens morphogenesis in camera-type eye", "Protein localization to endosome", and "Regulation of protein sumoylation". We also identified the top ten cellular components of GO including "Mitochondrial protein-containing complex", "Inner mitochondrial membrane protein complex", "Mitochondrial respirasome", "Respiratory chain complex", "Respirasome", "Oxidoreductase complex", "Mitochondrial respiratory chain complex III", "Respiratory chain complex III", "Endocytic vesicle lumen", and "Endoplasmic reticulum quality control compartment". We identified the top ten molecular functions of GO including "Transcription coregulator activity", "Cadherin binding", "Unfolded protein binding", "Proton transmembrane transporter activity", "Protein phosphatase binding”, "Transcription corepressor activity", "Phosphatase binding", "SMAD binding”, "Amyloid-beta binding", and "Beta-catenin binding".

\section{PPI network construction}

To further determine the relationship of the DEGs, we constructed the PPI network by String network and Cytoscape tool as described ${ }^{17-19}$. The combined score was set $>0.2$ as a cutoff to construct the PPI network by using the 48 nodes and 32 edges. Table 2 showed the top ten molecules with the highest degree scores. The top two cluster modules were indicated in Figure 3. We further analyzed the DEGs and the PPI network by Reactome (Figure 4 and Supplemental Table S1). We also determined the top ten significant biological processes through Reactome map including "ATF6 (ATF6-alpha) activates chaperone genes", "ATF6 (ATF6-alpha) activates chaperones", "Respiratory electron transport, ATP synthesis by chemiosmotic coupling, and heat production by uncoupling proteins", "Formation of ATP by chemiosmotic coupling", "Response of EIF2AK1 (HRI) to heme deficiency", "Cristae formation”, "The citric acid (TCA) cycle and respiratory electron transport", "Unfolded Protein Response (UPR)", and "Cellular responses to stress".

\section{Discussion}

miRNAs play key roles in tumor development but are aberrantly expressed in cancers such as breast cancers. Feng Tang et al found miR-125b was positively correlated with the migratory ability of breast cancer cells by using gain and loss-of-function methods ${ }^{20}$. However, the exact mechanisms of miR-125b treated breast cancer cells are still unknown.

The KEGG and GO analyses showed the mitochondria and endoplasmic reticulum oxidative phosphorylation pathways are important components in the miR-125b mediated breast cancer cells. Pamela Becherini et al found Sirtuin 6 (SIRT6) increases oxidative phosphorylation in breast cancer and enhances tumorigenesis in vivo study ${ }^{21}$. Isabelle Duroux-Richard et al found miR-125b regulates monocyte adaptation via mitochondrial metabolism and dynamics ${ }^{22}$. Interestingly, mitochondria metabolism involves various biological processes ${ }^{23-26}$, which is an effective target for cancer. Reprogramming metabolic pathways could ameliorate the metabolic inhibitors to suppress cancers, such as triple-negative breast cancer ${ }^{27}$. The endoplasmic reticulum (ER) attributes to the maintenance of cellular functions including organelle and lipogenesis, which plays essential roles in the progression of 
cancer $^{28-30}$. Milad Ashrafizadeh et al also found the autophagy and ER stress are promising methods to treat breast cancer ${ }^{31}$.

Base on the findings of KEGG and GO, we also identified ten significant molecules that involve in the miR$125 \mathrm{~b}$ mediated breast cancer. UQCRQ was identified as the significant protein in triple-negative breast cancer $^{32}$. Eduardo Cruz-Ramos et al found the CALR levels were increased in breast cancer cells lacking estrogen receptor alpha $(\mathrm{ERa})$, in comparison with those that express $\mathrm{Era}^{33}$. G-protein-coupled receptors (GPCRs) and Regulators of $\mathrm{G}$ protein signaling (RGS) are key regulators that modulate a variety of human diseases including arthritis, bone, and heart diseases ${ }^{34-43}$. Interestingly, calreticulin has been found to interact with several GPCR proteins such as AT1R and V2R $R^{44}$. Jiawei Zhou et al found HNRNPU enhances epithelial-mesenchymal transition, invasion capacity but represses the viability of invasive breast carcinoma cells ${ }^{45}$. Circadian gene clocks and their target genes are involved in a number of cell functions including proliferation, differentiation, metabolism, migration, and secretion ${ }^{46-50}$. Beibei Lai et al found HNRNPU is expressed in a circadian manner in the suprachiasmatic nucleus, which can be regulated by the circadian genes ${ }^{51}$. Penn Muluhngwi et al found ATP5G1 was identified as a target of miRNA in breast cancer $^{52}$. A study by Radhakrishnan Vishnubalaji found that the lack of function of NDUFB11 repressed the triple-negative breast cancer and enhanced its sensitivity to paclitaxe ${ }^{53}$. Kjerstin M Owens et al found UQCRH was increased in human breast cancers ${ }^{54}$. Thomas R Cawthorn et al found HSP90B1 is related to breast cancer metastasis by performing the proteomic analyses ${ }^{55}$. TGOLN2 and SAP18 were found to be closely related to the miRNA treatment in normal or tumor tissues ${ }^{56,57}$. Jianwei Lin et al found the increased XPOT showed a poor prognosis in patients with breast cancer ${ }^{58}$.

In conclusion, this study showed the impact of miR-125b on breast cancer. The mitochondria and endoplasmic reticulum oxidative phosphorylation pathways are mainly processes in miR-125b mediated breast cancer. Therefore, this study may be beneficial in the treatment of breast cancer by using miR$125 b$.

\section{Declarations}

\section{Author Contributions}

Hongmei Guo, Wei Zhang, Guangqi Liu, Tingxiang Chang: Methodology. Hanming Gu: Conceptualization, Writing and Editing.

\section{Funding}

This work was not supported by any funding. 


\section{Declarations of interest}

There is no conflict of interest to declare.

\section{References}

1. Rivenbark AG, O'Connor SM, Coleman WB: Molecular and cellular heterogeneity in breast cancer: challenges for personalized medicine. Am J Pathol 2013, 183:1113-24.

2. Schirrmacher V: From chemotherapy to biological therapy: A review of novel concepts to reduce the side effects of systemic cancer treatment (Review). Int J Oncol 2019, 54:407-19.

3. Sciaraffa T, Guido B, Khan SA, Kulkarni S: Breast cancer risk assessment and management programs: A practical guide. Breast J 2020, 26:1556-64.

4. Djulbegovic B, Kumar A, Soares HP, Hozo I, Bepler G, Clarke M, Bennett CL: Treatment success in cancer: new cancer treatment successes identified in phase 3 randomized controlled trials conducted by the National Cancer Institute-sponsored cooperative oncology groups, 1955 to 2006 . Arch Intern Med 2008, 168:632-42.

5. Valastyan S, Weinberg RA: Tumor metastasis: molecular insights and evolving paradigms. Cell 2011, 147:275-92.

6. Shrestha A, Martin C, Burton M, Walters S, Collins K, Wyld L: Quality of life versus length of life considerations in cancer patients: A systematic literature review. Psychooncology 2019, 28:1367-80.

7. Moazed D: Small RNAs in transcriptional gene silencing and genome defence. Nature 2009, 457:413-20.

8. Han J, Lee Y, Yeom KH, Kim YK, Jin H, Kim VN: The Drosha-DGCR8 complex in primary microRNA processing. Genes Dev 2004, 18:3016-27.

9. Macfarlane LA, Murphy PR: MicroRNA: Biogenesis, Function and Role in Cancer. Curr Genomics 2010, 11:537-61.

10. Slezak-Prochazka I, Durmus S, Kroesen BJ, van den Berg A: MicroRNAs, macrocontrol: regulation of miRNA processing. RNA 2010, 16:1087-95.

11. Bartel DP: MicroRNAs: target recognition and regulatory functions. Cell 2009, 136:215-33.

12. Oliveto $\mathrm{S}$, Mancino M, Manfrini N, Biffo S: Role of microRNAs in translation regulation and cancer. World J Biol Chem 2017, 8:45-56.

13. Wang Y, Zeng G, Jiang Y: The Emerging Roles of miR-125b in Cancers. Cancer Manag Res 2020, 12:1079-88.

14. Statello L, Guo CJ, Chen LL, Huarte M: Gene regulation by long non-coding RNAs and its biological functions. Nat Rev Mol Cell Biol 2021, 22:96-118.

15. Yu G, Wang LG, Han Y, He QY: clusterProfiler: an R package for comparing biological themes among gene clusters. OMICS 2012, 16:284-7. 
16. Li J, Wang W, Gu H: Identification of biological processes and signaling pathways for the knockout of REV-ERB in mouse brain. bioRxiv 2021:2021.11.22.469579.

17. Zhang $\mathrm{M}$, Wang J, Gu H: Identification of biological processes and signaling pathways in the stretched nucleus pulposus cells. bioRxiv 2021:2021.11.23.469730.

18. Hanming G, Wei W, Gongsheng Y: Identification of potential biomarkers and inhibitors in SARS-CoV-2 infected macaques. Research Square 2020.

19. Gu H, Wang W, Yuan G: Identification of biomarkers and candidate inhibitors for multiple myeloma. bioRxiv 2021:2021.02.25.432847.

20. Tang F, Zhang R, He Y, Zou M, Guo L, Xi T: MicroRNA-125b induces metastasis by targeting STARD13 in MCF-7 and MDA-MB-231 breast cancer cells. PLoS ONE 2012, 7:e35435.

21. Becherini P, Caffa I, Piacente F, Damonte P, Vellone VG, Passalacqua M, Benzi A, Bonfiglio T, Reverberi D, Khalifa A, Ghanem M, Guijarro A, Tagliafico L, Sucameli M, Persia A, Monacelli F, Cea M, Bruzzone S, Ravera S, Nencioni A: SIRT6 enhances oxidative phosphorylation in breast cancer and promotes mammary tumorigenesis in mice. Cancer Metab 2021, 9:6.

22. Duroux-Richard I, Roubert C, Ammari M, Presumey J, Grun JR, Haupl T, Grutzkau A, Lecellier CH, Boitez V, Codogno P, Escoubet J, Pers YM, Jorgensen C, Apparailly F: miR-125b controls monocyte adaptation to inflammation through mitochondrial metabolism and dynamics. Blood 2016, 128:3125-36.

23. Yuan G: Identification of biomarkers and pathways of mitochondria in sepsis patients. bioRxiv 2021:2021.03.29.437586.

24. Xu L, Cheng Q, Hua B, Cai T, Lin J, Yuan G, Yan Z, Li X, Sun N, Lu C, Qian R: Circadian gene Clock regulates mitochondrial morphology and functions by posttranscriptional way. bioRxiv 2018:365452.

25. Yuan G, Yang S, Liu M, Yang S: RGS12 is required for the maintenance of mitochondrial function during skeletal development. Cell Discov 2020, 6:59.

26. Cai T, Hua B, Luo D, Xu L, Cheng Q, Yuan G, Yan Z, Sun N, Hua L, Lu C: The circadian protein CLOCK regulates cell metabolism via the mitochondrial carrier SLC25A10. Biochim Biophys Acta Mol Cell Res 2019, 1866:1310-21.

27. Lee J, Yesilkanal AE, Wynne JP, Frankenberger C, Liu J, Yan J, Elbaz M, Rabe DC, Rustandy FD, Tiwari P, Grossman EA, Hart PC, Kang C, Sanderson SM, Andrade J, Nomura DK, Bonini MG, Locasale JW, Rosner MR: Effective breast cancer combination therapy targeting BACH1 and mitochondrial metabolism. Nature 2019, 568:254-8.

28. Wang $Y$, Wang $K$, Jin $Y$, Sheng $X$ : Endoplasmic reticulum proteostasis control and gastric cancer. Cancer Lett 2019, 449:263-71.

29. Yuan G, Hua B, Cai T, Xu L, Li E, Huang Y, Sun N, Yan Z, Lu C, Qian R: Clock mediates liver senescence by controlling ER stress. Aging 2017, 9:2647-65.

30. Fan XF, Wang XR, Yuan GS, Wu DH, Hu LG, Xue F, Gong YS: [Effect of safflower injection on endoplasmic reticulum stress-induced apoptosts in rats with hypoxic pulmonary hypertension]. Zhongguo Ying Yong Sheng Li Xue Za Zhi 2012, 28:561-7. 
31. Ashrafizadeh M, Mohammadinejad R, Tavakol S, Ahmadi Z, Sahebkar A: New Insight into TripleNegative Breast Cancer Therapy: The Potential Roles of Endoplasmic Reticulum Stress and Autophagy Mechanisms. Anticancer Agents Med Chem 2021, 21:679-91.

32. He J, Yang J, Chen W, Wu H, Yuan Z, Wang K, Li G, Sun J, Yu L: Molecular Features of Triple Negative Breast Cancer: Microarray Evidence and Further Integrated Analysis. PLoS ONE 2015, 10:e0129842.

33. Cruz-Ramos E, Sandoval-Hernandez A, Tecalco-Cruz AC: Differential expression and molecular interactions of chromosome region maintenance 1 and calreticulin exportins in breast cancer cells. $J$ Steroid Biochem Mol Biol 2019, 185:7-16.

34. Yuan G, Huang Y, Yang S-t, Ng A, Yang S: RGS12 inhibits the progression and metastasis of multiple myeloma by driving M1 macrophage polarization and activation in the bone marrow microenvironment. Cancer Commun, n/a.

35. Yuan G, Fu C, Yang ST, Yuh DY, Hajishengallis G, Yang S: RGS12 Drives Macrophage Activation and Osteoclastogenesis in Periodontitis. J Dent Res 2021:220345211045303.

36. Fu C, Yuan G, Yang ST, Zhang D, Yang S: RGS12 Represses Oral Cancer via the Phosphorylation and SUMOylation of PTEN. J Dent Res 2020:22034520972095.

37. Mao SZ, Fan XF, Xue F, Chen R, Chen XY, Yuan GS, Hu LG, Liu SF, Gong YS: Intermedin modulates hypoxic pulmonary vascular remodeling by inhibiting pulmonary artery smooth muscle cell proliferation. Pulm Pharmacol Ther 2014, 27:1-9.

38. Yuan G, Yang S, Gautam M, Luo W, Yang S: Macrophage regulator of G-protein signaling 12 contributes to inflammatory pain hypersensitivity. Ann Transl Med 2021, 9:448.

39. Yuan G, Yang S, Yang S: Macrophage RGS12 contributes to osteoarthritis pathogenesis through enhancing the ubiquitination. Genes \& Diseases 2021.

40. Hanming G: nuotrophils arthritis. Research Square 2021.

41. Yuan G, Yang S, Yang S, Ng A, Oursler MJ: RGS12 is a critical proinflammatory factor in the pathogenesis of inflammatory arthritis via acting in Cox2-RGS12-NF kappa B pathway activation loop. J Bone Miner Res: WILEY 111 RIVER ST, HOBOKEN 07030-5774, NJ USA, 2019. pp. 147-.

42. Sun L, Ye RD: Role of G protein-coupled receptors in inflammation. Acta Pharmacol Sin 2012, 33:342-50.

43. Yuan G, Yang S, Ng A, Fu C, Oursler MJ, Xing L, Yang S: RGS12 Is a Novel Critical NF-kappaB Activator in Inflammatory Arthritis. iScience 2020, 23:101172.

44. Dong C, Filipeanu CM, Duvernay MT, Wu G: Regulation of G protein-coupled receptor export trafficking. Biochim Biophys Acta 2007, 1768:853-70.

45. Zhou J, Guo Y, Huo Z, Xing Y, Fang J, Ma G, Han Q, Wang M, Xu Q: Identification of therapeutic targets and prognostic biomarkers from the hnRNP family in invasive breast carcinoma. Aging 2021, 13:4503-21.

46. Zhu Z, Hua B, Xu L, Yuan G, Li E, Li X, Sun N, Yan Z, Lu C, Qian R: CLOCK promotes 3T3-L1 cell proliferation via Wnt signaling. IUBMB Life 2016, 68:557-68. 
47. Yuan G, Hua B, Yang Y, Xu L, Cai T, Sun N, Yan Z, Lu C, Qian R: The Circadian Gene Clock Regulates Bone Formation Via PDIA3. J Bone Miner Res 2017, 32:861-71.

48. Zhu Z, Hua B, Shang Z, Yuan G, Xu L, Li E, Li X, Sun N, Yan Z, Qian R, Lu C: Altered Clock and Lipid Metabolism-Related Genes in Atherosclerotic Mice Kept with Abnormal Lighting Condition. Biomed Res Int 2016, 2016:5438589.

49. Yuan G, Xu L, Cai T, Hua B, Sun N, Yan Z, Lu C, Qian R: Clock mutant promotes osteoarthritis by inhibiting the acetylation of NFkappaB. Osteoarthritis Cartilage 2019, 27:922-31.

50. Zhu Z, Xu L, Cai T, Yuan G, Sun N, Lu C, Qian R: Clock represses preadipocytes adipogenesis via GILZ. J Cell Physiol 2018, 233:6028-40.

51. Lai B, Zou J, Lin Z, Qu Z, Song A, Xu Y, Gao X: Haploinsufficiency of hnRNP U Changes Activity Pattern and Metabolic Rhythms. Am J Pathol 2018, 188:173-83.

52. Muluhngwi P, Alizadeh-Rad N, Vittitow SL, Kalbfleisch TS, Klinge CM: The miR-29 transcriptome in endocrine-sensitive and resistant breast cancer cells. Sci Rep 2017, 7:5205.

53. Vishnubalaji R, Alajez NM: Transcriptional landscape associated with TNBC resistance to neoadjuvant chemotherapy revealed by single-cell RNA-seq. Mol Ther Oncolytics 2021, 23:151-62.

54. Owens KM, Kulawiec M, Desouki MM, Vanniarajan A, Singh KK: Impaired OXPHOS complex III in breast cancer. PLoS ONE 2011, 6:e23846.

55. Cawthorn TR, Moreno JC, Dharsee M, Tran-Thanh D, Ackloo S, Zhu PH, Sardana G, Chen J, Kupchak P, Jacks LM, Miller NA, Youngson BJ, lakovlev V, Guidos CJ, Vallis KA, Evans KR, McCready D, Leong WL, Done SJ: Proteomic analyses reveal high expression of decorin and endoplasmin (HSP90B1) are associated with breast cancer metastasis and decreased survival. PLoS ONE 2012, 7:e30992.

56. Yang H, Su H, Hu N, Wang C, Wang L, Giffen C, Goldstein AM, Lee MP, Taylor PR: Integrated analysis of genome-wide miRNAs and targeted gene expression in esophageal squamous cell carcinoma (ESCC) and relation to prognosis. BMC Cancer 2020, 20:388.

57. Farhana L, Dawson MI, Fontana JA: Down regulation of miR-202 modulates Mxd1 and Sin3A repressor complexes to induce apoptosis of pancreatic cancer cells. Cancer Biol Ther 2015, 16:11524.

58. Lin J, Hou Y, Huang S, Wang Z, Sun C, Wang Z, He X, Tam NL, Wu C, Wu L: Exportin-T promotes tumor proliferation and invasion in hepatocellular carcinoma. Mol Carcinog 2019, 58:293-304.

\section{Tables}

Tables 1-2 are in the supplementary files section.

\section{Figures}


Heatmap and volcano plot were obtained from the low and high expression of miR-125b in breast cancer cells

(A) Heatmap of significant differentially expressed genes (DEGs). Significant DEGs $(P<0.01)$ were used to create the heatmap.

(B) Volcano plot for DEGs from the low and high expression of miR-125b in breast cancer cells. The mostly changed genes are highlighted by grey dots.

\section{Figure 2}

KEGG and GO analyses of DEGs from the low and high expression of miR-125b in breast cancer cells

(A) KEGG analysis, (B) Biological processes, (C) Cellular components, (D) Molecular functions.

A

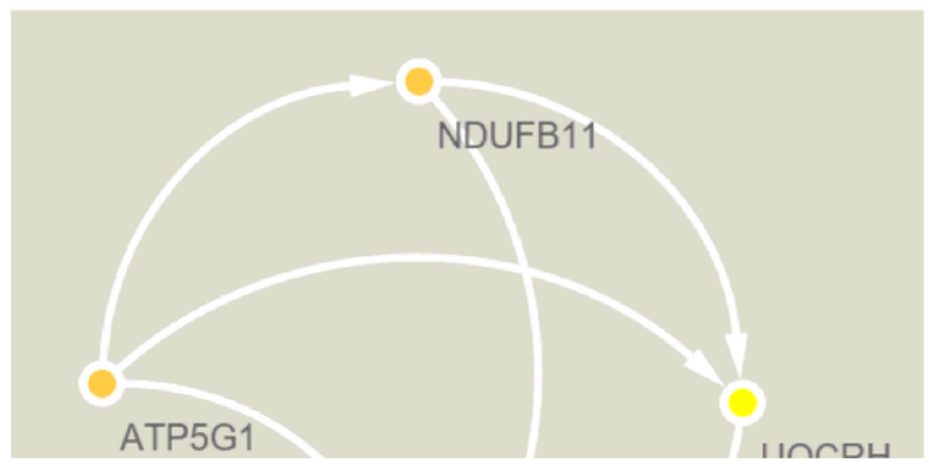

B

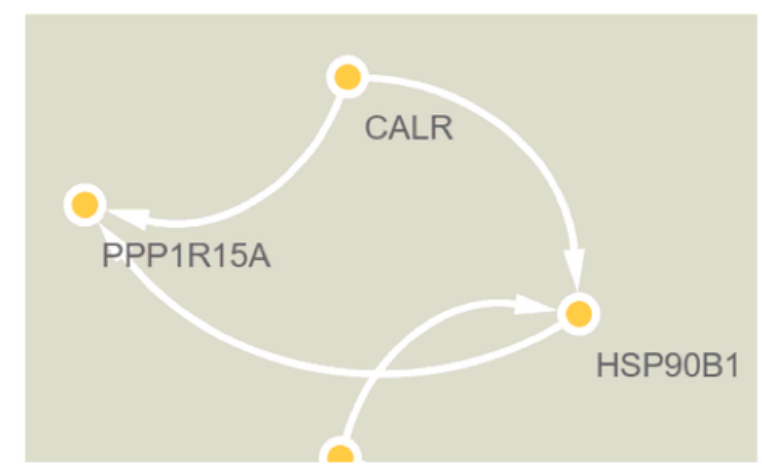

Figure 3

The PPI network analysis of DEGs from the low and high expression of miR-125b in breast cancer cells

Cluster 1 (A) and cluster 2 (B) were the top two clusters and were constructed by MCODE in Cytoscape.

Figure 4 
Reactome map representation of the significant biological processes from the low and high expression of miR-125b in breast cancer cells

\section{Supplementary Files}

This is a list of supplementary files associated with this preprint. Click to download.

- SupplementalTableS1.xlsx

- Tables.docx 